\title{
A Numerical Study on Jet Structure and Noise Emission of Underexpanded Radial Jet
}

\author{
Takumi Nojima ${ }^{1}$, Yoko Sakakibara ${ }^{2}$, Masaki Endo² $^{2}$, Hiromasa Suzuki ${ }^{3}$ \\ ${ }^{1}$ Graduate School of Science and Engineering, Tokyo Denki University, Saitama, Japan \\ ${ }^{2}$ Division of Mechanical Engineering, Tokyo Denki University, Saitama, Japan \\ ${ }^{3}$ Tokyo Metropolitan College of Industrial Technology, Tokyo, Japan \\ Email: 17rmt15@ms.dendai.ac.jp
}

How to cite this paper: Nojima, T., Sakakibara, Y., Endo, M. and Suzuki, H. (2019) A Numerical Study on Jet Structure and Noise Emission of Underexpanded Radial Jet. Open Journal of Fluid Dynamics, 9, 82-91

https://doi.org/10.4236/ojfd.2019.91006

Received: February 10, 2019

Accepted: March 11, 2019

Published: March 14, 2019

Copyright $\odot 2019$ by author(s) and Scientific Research Publishing Inc. This work is licensed under the Creative Commons Attribution International License (CC BY 4.0).

http://creativecommons.org/licenses/by/4.0/

\begin{abstract}
In this study, an underexpanded radial jet issuing from a small gap between two circular tubes facing each other is investigated numerically. Radial jet is formed, for example, downstream of high-pressure valves in piping system and of poppet valves in engines, and causes many industrial problems such as the noise generation and the fatigue failure of structure. In this study, the jet issuing from a small gap between two tubes with same diameter is numerically simulated. The flow field is assumed to be axisymmetric against the central axis of tubes and to be symmetric against the intermediate plane between the exits of two tubes. The axisymmetric Euler equations are solved using symmetric TVD (Total Variation Diminishing) scheme. The effects of nozzle pressure ratio and of diameter of circular tubes on the structure and the behavior of jets are examined. Typical cell structure of underexpanded jet appears in radial jet and the length of cell becomes smaller in downstream region because the jet spreads radially like a disc. The length and width of first cell are larger with higher nozzle pressure ratio. Many vortices are generated one after another near the jet boundary and move downstream, which cause the oscillation of jet. Outside of jet, two types of density waves are observed. One of them propagates toward the nozzle (toward the upstream region) and the other propagates in opposite direction. Focusing on the pressure change caused by the former waves, which is related to well-known screech, dominant frequency obtained by FFT analysis was found to become lower with higher pressure ratio and smaller diameter of tube.
\end{abstract}

\section{Keywords}

Compressible Flow, Screech, Oscillation, Underexpanded Jet 


\section{Introduction}

Supersonic jet is used in various industrial scenes, such as an assist gas to remove molten material in laser processing [1], a cooling jet in glass tempering process [2] and so on. The jet issuing from a convergent nozzle becomes underexpanded when the pressure ratio across the nozzle is higher than the critical pressure ratio (about 1.893 in case of the air). The underexpanded jet has typical cellular structure in barrel shape, which consists of the alternate expansion and compression regions. Shock waves appear in such a cell structure. Powell [3] reports the screech sound caused by the interaction between these shock waves and the disturbances traveling around the jet. It is well known that the screech is the narrow-band noise with high frequency and it has multiple modes. Tam [4] examined the generation mechanism and the direction of screech in case of the jet issuing from a circular nozzle. And it has shown that the Mach number and the size of cell affect the characteristics of the screech, by comparison with Norum's experimental results [5]. Besides, so far, two-dimensional rectangular jets and axisymmetric jets have been studied by many researchers concerning jet structure, oscillation phenomena, noise generation and so on [6]-[11].

The present study focuses on the underexpanded jet which is radially discharged into the atmosphere. The radial jet issuing from a small gap between two circular tubes facing each other is numerically investigated and the flow characteristics are shown, such as the jet structure and the relation between oscillation phenomena and noise emission. The radial underexpanded jet is formed, for example, downstream of valves in high-pressure piping system and of poppet valves in engines. Also the wall jet of impinging jet has similar structure when axisymmetric supersonic jet impinges on a flat plate. In such flow field, the oscillation of jet and the noise generation cause some problems in industrial point of view [12].

\section{Numerical Scheme}

To examine the characteristics of underexpanded radial jet in this study, the flow field that Kawasaki [13] experimentally studied is simplified and it is devised as a computational model as shown in Figure 1. Two circular tubes each fitted to a high pressure tank, are installed face to face each other. The airflows through tubes collide with each other on the intermediate plane between two tubes, change their directions and radially spread out into the atmosphere from a gap of tubes. The inside wall of each tube near the gap is designed for the air to reach the sonic speed at the exit of gap. In the figure, the shapes of jet on the cross sections along seven radial directions are drawn with black dashed line as examples. The computational model is more simplified, so that the flow field is assumed to be symmetric against the intermediate plane between two tubes and to be axisymmetric against the centerline of tube.

Figure 2 shows the computational model used in this study, which corresponds to the region surrounded by a red dotted line in Figure 1. The central axis 
of tubes is taken as $x$-axis, and $y$-axis is taken to be normal to $x$-axis as shown in this figure. The origin of this coordinate system is on the intermediate plane between tubes. Because of the symmetric conditions as mentioned above, only the positive region of $y$ is taken as the computational domain. The jet is exhausted from gap $b$ into the atmosphere at speed of sound. Therefore, the critical conditions are applied to the nozzle exit as the boundary conditions. The outer wall of the tube is the slip wall and the symmetric conditions are given on the left-side boundary of computational domain. Because the upper-side and the right-side boundaries are located in the atmosphere, the atmospheric pressure is given and the velocity and the density are calculated using the method of characteristics on the boundaries.

Computational parameters are shown in Table 1. In this study, two types of circular tube are used. Outer diameter of the one is $D=12 \mathrm{~mm}$ and that of the other $D=16 \mathrm{~mm}$. The gap between the tubes $b=2 \mathrm{~mm}$ is fixed. Compressed air from two tubes collides with each other, changes the direction and issues from the gap into the atmosphere. In this study, the nozzle, which consists of two tubes, discharging the underexpanded jet is called "slit nozzle".

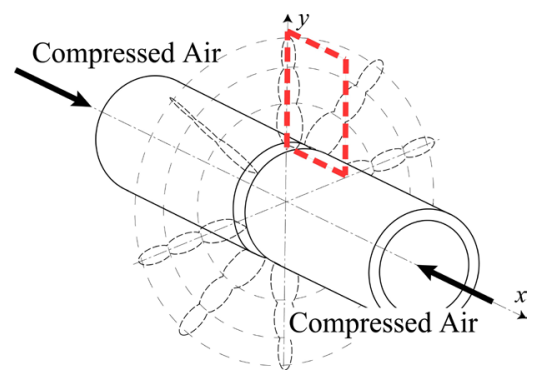

Figure 1. Slit nozzle.

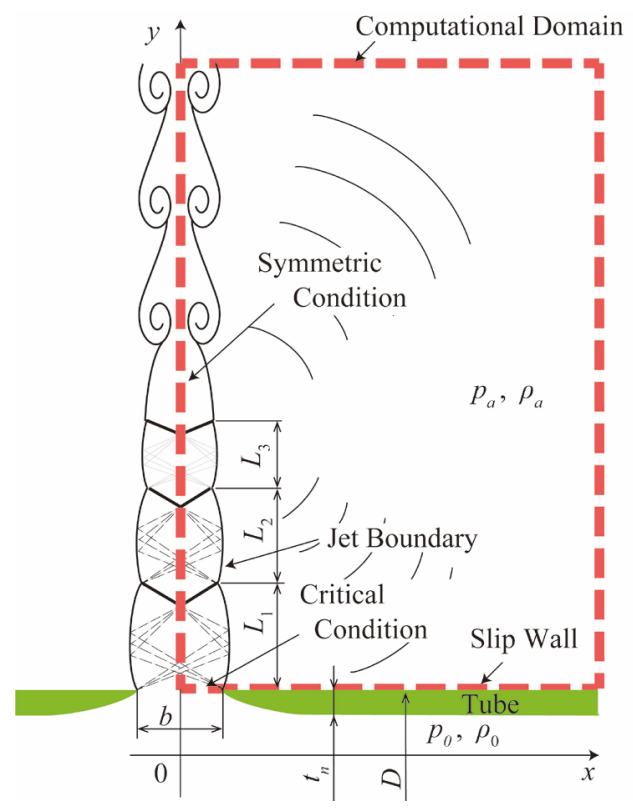

Figure 2. Computational model. 
Table 1. Computational parameters.

\begin{tabular}{cc}
\hline Outer diameter of tube $D$ & $12 \mathrm{~mm}, 16 \mathrm{~mm}$ \\
\hline Nozzle width $b$ & $2 \mathrm{~mm}$ \\
Tube thickness $t$ & $1 \mathrm{~mm}$ \\
Pressure Ratio $P R$ & $2.0-6.0$ \\
\hline
\end{tabular}

The region surrounded by a red dashed line in Figure 2 is the computational domain. 300 computational grids are uniformly taken between $x / b=0.0$ and 7.5 in $x$-direction and 600 grids between nozzle exit and $y / b=15.0$ are taken in $y$-direction. The grids outside of this uniform region become coarser both in $x$ and $y$-directions as further away from the origin. The maximum value of computational area is $x / b=11.0$ and $y / b=49.5$. The jet is exhausted from lower left of computational domain, and the air flows toward the upper side of the figure. Pressure ratio $P R$ which is the ratio of the pressure in high pressure chamber $p_{0}$ and the atmospheric pressure $p_{\mathrm{a}}$ is changed between 2.0 to 6.0.

As mentioned above, the flow field is assumed to be axisymmetric against $x$-axis, and to be symmetric against the intermediate plane between two tubes. Therefore, the axisymmetric Euler equations are solved using our in-house program with the symmetric TVD (Total Variation Diminishing) scheme [14], which has second order accuracy both in space and time and has been shown to work well on the analysis of the impinging-jet simulation [15].

\section{Results and Discussion}

\subsection{Pressure and Density Contours}

Figure 3 show the instantaneous jet structure obtained by numerical simulation. The jet is exhausted from the nozzle exit in the bottom of the figure. Outer diameter of slit nozzle is $D=12 \mathrm{~mm}$. The left half of figure shows the pressure contours and the right half the density contours.

In Figure 3(a), two barrel-shaped cells are formed just downstream of nozzle exit and the vortices dominate in the downstream region. The width and the length of second cell are found to be smaller than those of first cell. This is conspicuous feature of radial jet because the jet spreads radially and then, decrease ratio of the neighboring cell sizes is larger than that of the rectangular jet and the axisymmetric jet. In Figures 3(b)-(d), the similar jet structure is found, while the length of first cell becomes larger at higher pressure ratio.

Surrounding the jet, two types of density waves can be observed. One of them propagates toward the nozzle (toward the upstream region) and the other propagates in opposite direction. In case of the jet with $D=12 \mathrm{~mm}$, frequency of pressure change at fixed point near the nozzle, which is caused by the density waves propagating upstream, was reported to agree with that of screech tone obtained by experiment [16].

Figure 4 show the instantaneous jet structure with $D=16 \mathrm{~mm}$ for different $P R$. Although the jet structure and density waves show the almost same tendency 
as those in Figure 3, the size of cells is seen to become comparatively large. The radial jet narrows downstream to satisfy the continuity equation, while the rectangular jet does not change the jet width. The smaller the diameter becomes, the more the effect of the diameter on the jet width appears. Therefore, the jet with $D=12 \mathrm{~mm}$ becomes narrower than that with $D=16 \mathrm{~mm}$.

\subsection{Cell Length}

Figure 5 shows cell length of the jet for each pressure ratio measured from the density contours. $L_{1}, L_{2}$ and $L_{3}$ are the length of first, second and third cell, which are shown in Figure 2. The cell length of radial jet becomes longer as pressure ratio becomes higher, this is similar with well-known characteristics of the rectangular jet [17]. Although the cell length of the rectangular jet slightly decreases downstream because of the shearing stress in jet boundary, as notable characteristics of radial jet, the decrease ratio of adjacent cell sizes is higher than that of rectangular jet because of the effect which narrows the jet width as mentioned above. These are confirmed also in Figure 3 and Figure 4.

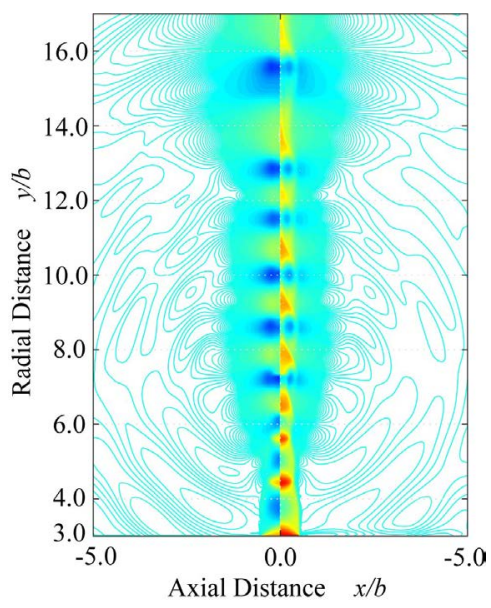

(a) $P R=3.0$

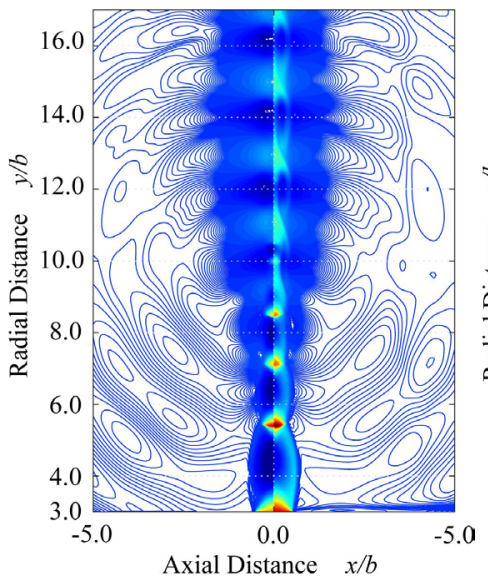

(c) $P R=5.0$

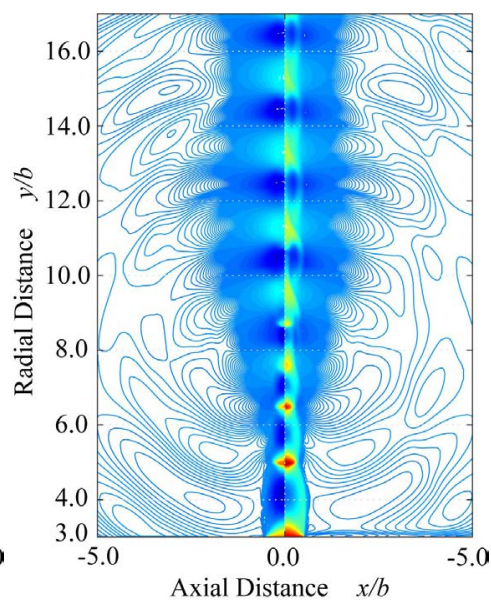

(b) $P R=4.0$

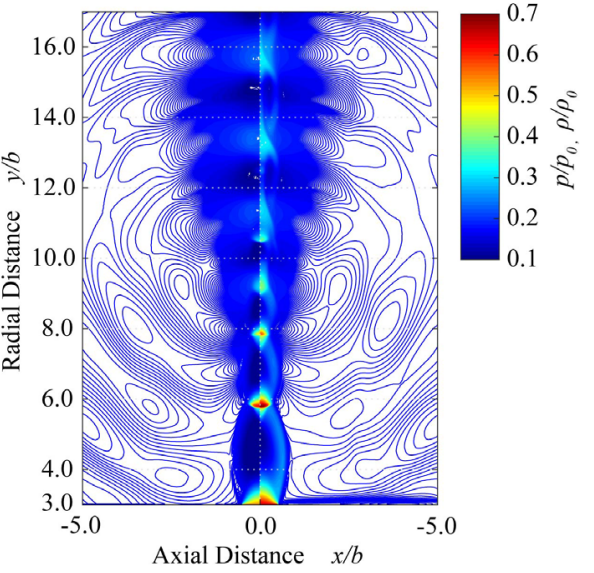

(d) $P R=6.0$

Figure 3. Pressure (left) and density (right) contours of underexpanded radial jet $(D=12 \mathrm{~mm})$. 


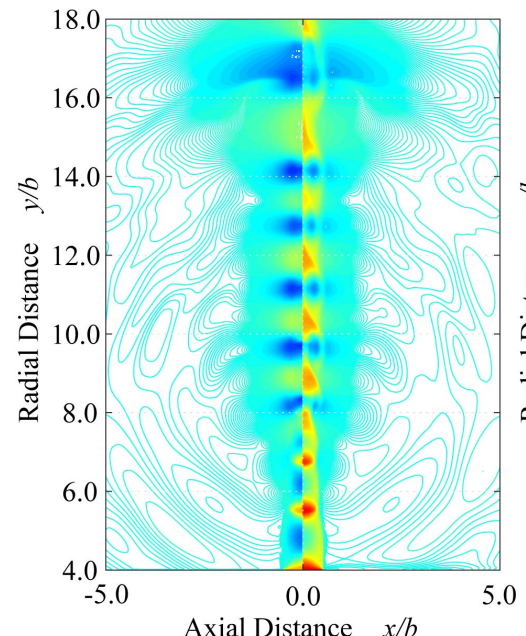

(a) $P R=3.0$

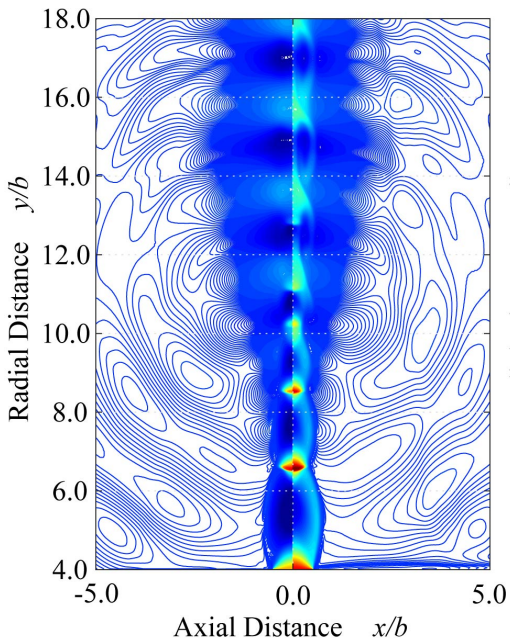

(c) $P R=5.0$

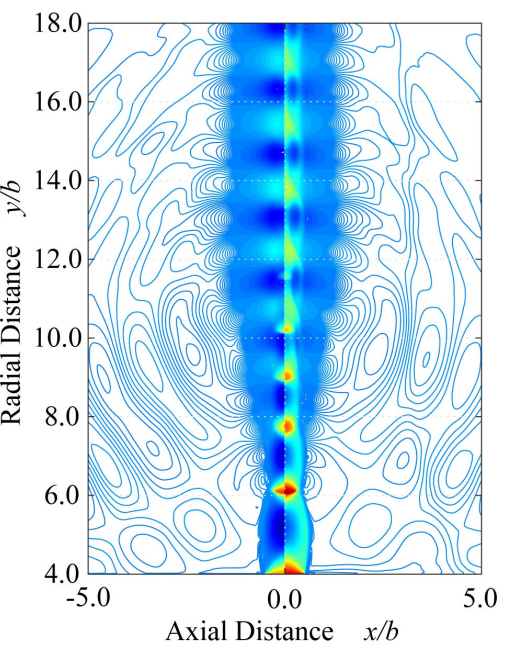

(b) $P R=4.0$

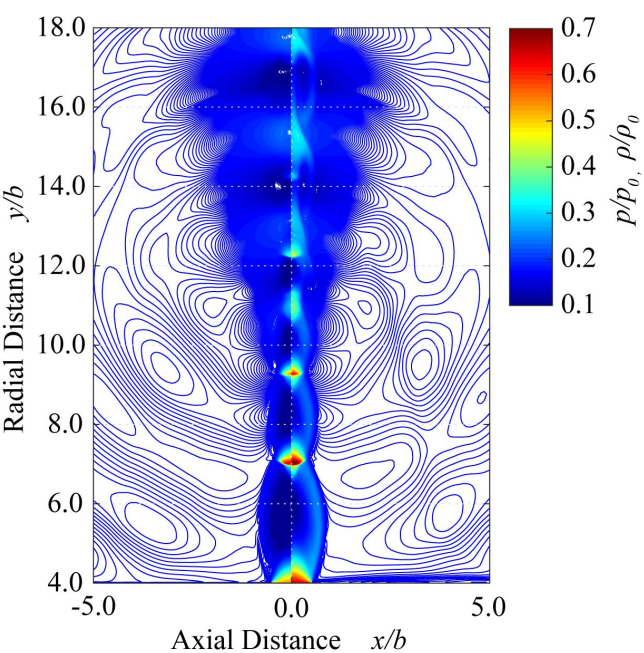

(d) $P R=6.0$

Figure 4. Pressure (left) and density (right) contours of underexpanded radial jet $(D=16$ $\mathrm{mm})$.

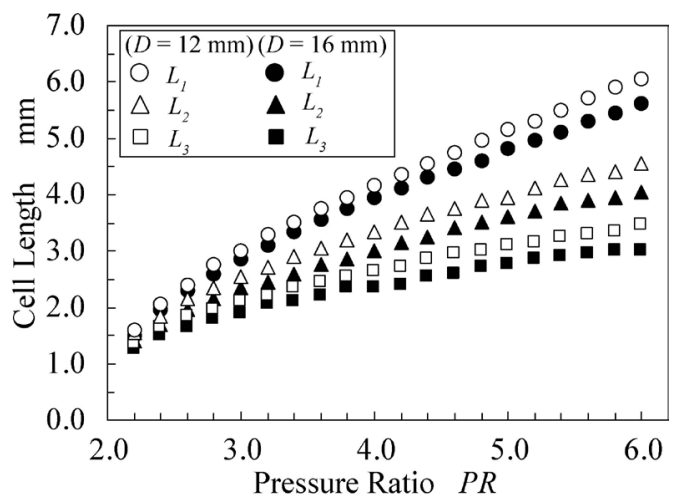

Figure 5. Cell length.

In Figure 5, when $P R$ is less than 2.8, cell length gradually increases and, when $P R$ is higher, it becomes larger linearly. Furthermore, as discussed above, 
the cell length with $D=16 \mathrm{~mm}$ is found to be larger than that with $D=12 \mathrm{~mm}$ because of the effect which narrows the jet width.

\subsection{Frequency of Jet Oscillation}

Radial jet oscillates periodically with vortices moving downstream. Figure 6 shows the example of the result of FFT analysis of pressure history recorded at the end of 2nd cell of the jet. This is the result for $D=12 \mathrm{~mm}$ at $P R=3.0$. Dominant frequency at about $80 \mathrm{kHz}$ appears, which can be considered to be screech tone because, in Figure 3, the cell node of 2nd cell is positioned in the region surrounded by density waves which propagate upstream toward the nozzle.

Figure 7 shows the screech frequency for different pressure ratios. Screech frequency tends to decrease when the pressure ratio increases. And it can be considered that there are several modes in screech frequency because jump to the other mode appears in several places. Such characteristic of the frequency has been reported by many researchers concerning the rectangular jet. And the frequency is well-known to be inversely proportional to the nozzle width. In case of the radial jet as shown in Figure 7, as a whole, the frequencies of $D=12 \mathrm{~mm}$ are larger than those of $D=16 \mathrm{~mm}$. In other words, for the radial jet with $D=12$ $\mathrm{mm}$ to have same frequencies as those with $D=16 \mathrm{~mm}$, the larger $P R$ is necessary than that of $D=16 \mathrm{~mm}$. For example, the frequency with $D=12 \mathrm{~mm}$ at $P R$ $=3.2$ is almost equal to that with $D=16 \mathrm{~mm}$ at $P R=2.8$. Thus, screech frequency depends on $D$ and $P R$.

The cell lengths $L_{1}, L_{2}$ and $L_{3}$ at almost same frequency are listed in Table 2. This table shows three cases that frequency is almost same. As the first case, the frequency with $D=12 \mathrm{~mm}$ at $P R=3.2$ is $78.2 \mathrm{kHz}$ and that with $D=16$ at $P R=$ 2.8 is $79.3 \mathrm{kHz}$. These frequencies are very close. Under these conditions, although the difference of length $L_{1}$ is 0.3 , the difference of $L_{2}$ is 0.1 and that of $L_{3}$ is also 0.1 . In the same manner, the frequencies of second case are $66.4 \mathrm{kHz}$ and $67.1 \mathrm{kHz}$ and those of third case are $54.0 \mathrm{kHz}$ and $54.4 \mathrm{kHz}$. Also in the second and the third case, the difference of $L_{2}$ and that of $L_{3}$ are 0.1 , respectively. Thus, the 2 nd or the 3 rd cell length is closer than that of 1st cell length in every case. So, it is considered that the screech frequency of jet depends on the length of $2 \mathrm{nd}$ or 3 rd cell in case of radial jet.

\subsection{Directivity of Density Waves}

In order to investigate the directivity of the screech of the radial jet, the sound pressure of screech surrounding the jet is also measured. Measurement is performed in a semicircle with 3rd cell end as a center. The radius of the semicircle is the distance between nozzle exit and 3rd cell end. Measurement is carried out at 10 positions stepped by 15 degrees. At every point, the pressure history is analyzed by FFT and screech frequency is obtained like Figure 6 . The sound pressure of screech is shown as a radar chart in Figure 8. The sound pressure 
becomes strongest at the position " 2 ", " 3 " and " 10 ". In addition the noise radiation cannot be seen in the direction of 90 degrees. These features are similar to axisymmetric jet [4].

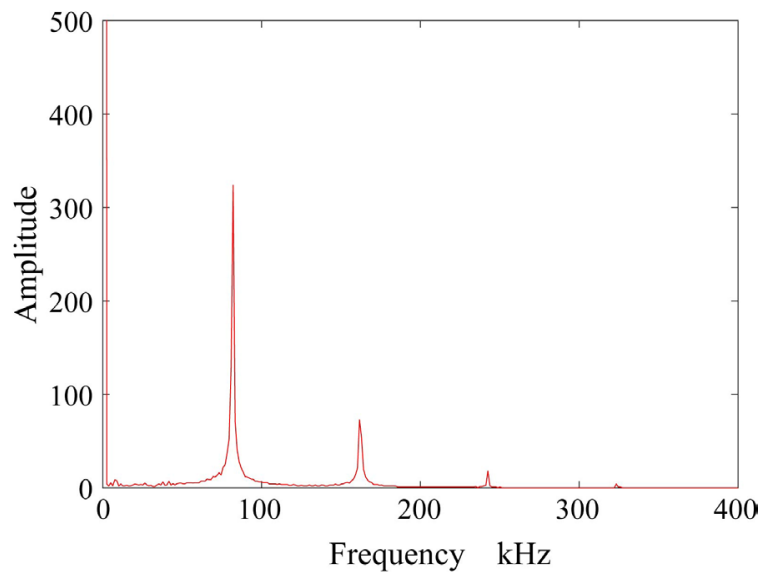

Figure 6. Analysis of pressure oscillation in jet.

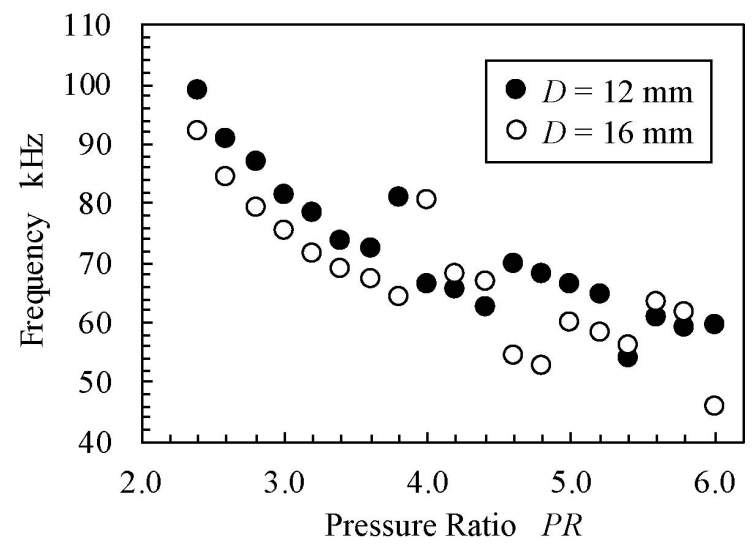

Figure 7. Screech frequency for different $P R$ and $D$.

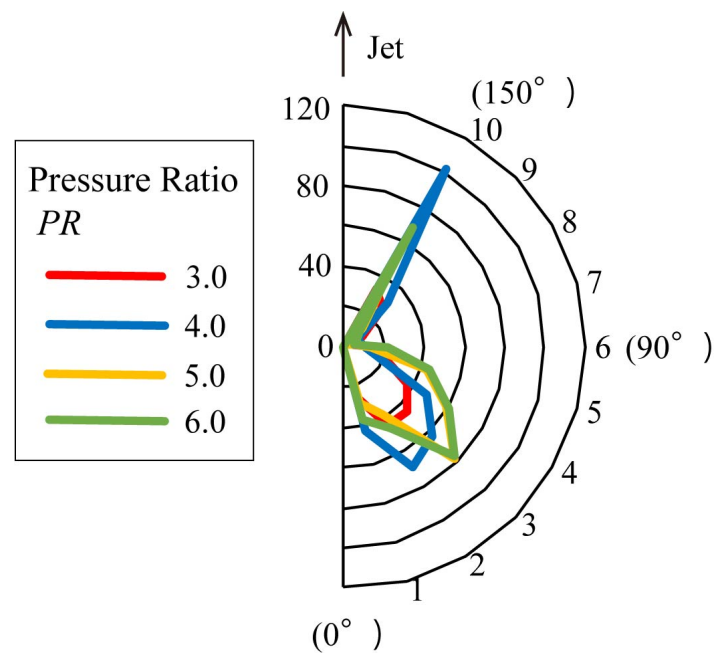

Figure 8. Sound pressure distribution $(D=12 \mathrm{~mm})$. 
Table 2. Screech frequency and cell length.

\begin{tabular}{cccccc}
\hline & & Frequency & \multicolumn{3}{c}{ Cell Length mm } \\
\cline { 3 - 6 }$D$ & $P R$ & $\mathrm{kHz}$ & $L_{1}$ & $L_{2}$ & $L_{3}$ \\
\hline 12 & 3.2 & 78.2 & 3.1 & 2.5 & 2.1 \\
16 & 2.8 & 79.3 & 2.8 & 2.4 & 2.0 \\
12 & 4.0 & 66.4 & 4.0 & 3.0 & 2.4 \\
16 & 3.6 & 67.1 & 3.8 & 3.1 & 2.5 \\
12 & 5.4 & 54.0 & 5.1 & 3.9 & 2.9 \\
16 & 4.6 & 54.4 & 4.8 & 3.8 & 3.0 \\
\hline
\end{tabular}

\section{Conclusions}

The underexpanded radial jet is numerically simulated and the flow characteristics of the jet are examined, especially concerning its cellular structure and the relation between the oscillation phenomena and the screech. Thereby, the following conclusions are drawn:

1) Cell length of first cell becomes larger with higher pressure ratio and decrease ratio of cell length of radial jet is larger than that of rectangular jet.

2) The radial jet issuing from slit nozzle with smaller diameter has higher frequency screech and the frequency decreases with higher pressure ratio.

3) Sound pressure of the noise emitted from the radial jet has directivity. Upstream-travelling noise forms screech phenomena.

4) Screech frequency mainly depends on the length of 2nd or 3rd cell.

In addition, the computational model presented in this paper can be considered useful for analyzing the behavior of the rectangular jet, because the configuration of jet becomes two-dimensional when the tube diameter tends to infinity. Therefore, to understand the characteristics of the radial jet will become one of clues to clarify those of the rectangular jet which has been studied for many years.

\section{Conflicts of Interest}

The authors declare no conflicts of interest regarding the publication of this paper.

\section{References}

[1] Kanaoka, M. and Kotou, S. (1993) Study of the Relationship between the Accuracy in $\mathrm{Co}_{2}$ Laser Cutting and the Assist Gas. Transactions of the Japan Society of Mechanical Engineers $C, 59,350-357$. (In Japanese) https://doi.org/10.1299/kikaic.59.1952

[2] Aratani, N., Narayanswami, N., Ojima, H. and Takayama, K. (1995) Studies of Supersonic Jets and Shock Waves Generated during Glass Tempering Process. Transactions of the Japan Society of Mechanical Engineers B, 61, 308-313. (In Japanese) https://doi.org/10.1299/kikaib.61.3706

[3] Powell, A. (1953) The Noise of Chocked Jets. The Journal of the Acoustical Society 
of America, 25, 385-389. https://doi.org/10.1121/1.1907052

[4] Tam, C.K.W. and Viswanathan, K. (2014) Harmonics of Jet Screech Tones. AIAA Journal, 52, 2471-2479. https://doi.org/10.2514/1.J052850

[5] Norum, T.D. (1983) Screech Suppression in Supersonic Jets. AIAA Journal, 21, 235-240. https://doi.org/10.2514/3.8059

[6] Beneddine, S., Mettot, C. and Sipp, D. (2015) Global Stability Analysis of Underexpanded Screeching Jets. European Journal of Mechanics B/Fluids, 49, 392-399. https://doi.org/10.1016/j.euromechflu.2014.05.006

[7] Ozawa, Y., Nonomura, T., Fujii, K., Yamamoto, M. and Mamori, Y. (2015) Experimental Study on the Velocity Distribution in the Supersonic Jet Shear Layerand the Acoustic Waves. Fluid Engineering Department Conference Proceeding of the Japan Society of Mechanical Engineers, November 2015, Tokyo, 1-3. (In Japanese)

[8] Jagadeesh, C., Gowree, E.R., Jadidbonab, H. and Atkin, C.J. (2018) On the Temporal Analysis of Acoustic Waves Using Schlieren Imaging. Measurement, 116, 507-513. https://doi.org/10.1016/j.measurement.2017.11.018

[9] Powell, A., Umeda, Y. and Ishii, R. (1992) Observation of the Oscillation Modes of Choked Circular Jets. The Journal of the Acoustical Society of America, 92, 2823-2836. https://doi.org/10.1121/1.404398

[10] Tam, C.K.W. and Burton, D. (1984) Sound Generated by Instability Waves of Supersonic Flows. Part 1. Two-Dimensional Mixing Layers. Journal of Fluid Mechanics, 138, 249-271. https://doi.org/10.1017/S0022112084000112

[11] Tam, C.K.W. and Burton, D. (1984) Sound Generated by Instability Waves of Supersonic Flows. Part 2. Axisymmetric Jets. Journal of Fluid Mechanics, 138, 273-295. https://doi.org/10.1017/S0022112084000124

[12] Akatsuka, J., Ogura, N., Homma, T., Watanabe, Y. and Kameda, M. (2017) Experimental Evaluation of Acoustic and Thrust Characteristics of Exhaust Nozzles Using a Model Engine. Aerospace Technology Japan, 16, 73-79. (In Japanese)

[13] Kawasaki, K., Suzuki, H., Endo, M. and Sakakibara, Y. (2018) A Study on Screech Tone Emitted from Underexpanded Radial Jet. Proceedings of the 7 th Asian Joint Workshop on Thermophysics and fluid Science 2018, Trivandrum, India, 21-24 November 2018, 269-274.

[14] Yee, H.C. (1987) Upwind and Symmetric Shock Capturing. NASA Technical Memorandum, 89464.

[15] Sakakibara, Y. and Iwamoto, J. (1998) Numerical Study of Oscillation Mechanism in Underexpanded Jet Impinging on Plate. Journal of Fluids Engineering, 120, 477-481. https://doi.org/10.1115/1.2820687

[16] Kawasaki, K., Hirano, T., Endo, M. and Sakakibara, Y. (2014) Noise Emission and Structure of Underexpanded Radial Jet. Proceedings of the 5 th Asian Joint Workshop on Thermophysics and Fluid Science 2014, Nagasaki, 23-26 September 2014, $1-8$.

[17] Powell, A. (1953) On The Mechanism of Choked Jet Noise. Proceedings of the Physical Society B, 66, 1039-1057. https://doi.org/10.1088/0370-1301/66/12/306 